\title{
Global Existence and Blow-Up of Solutions for Nonlinear Klein-Gordon Equation with Damping Term and Nonnegative Potentials
}

\author{
Wen-Yi Huang' and Wen-Li Chen ${ }^{2}$ \\ ${ }^{1}$ Department of Applied Mathematics, Southwestern University of Finance and Economics, Chengdu 610074, China \\ ${ }^{2}$ College of Mathematics and Software Science, Sichuan Normal University, Chengdu 610066, China \\ Correspondence should be addressed to Wen-Yi Huang; huangwy@swufe.edu.cn
}

Received 2 May 2014; Accepted 5 August 2014; Published 14 October 2014

Academic Editor: Adrian Petrusel

Copyright (c) 2014 W.-Y. Huang and W.-L. Chen. This is an open access article distributed under the Creative Commons Attribution License, which permits unrestricted use, distribution, and reproduction in any medium, provided the original work is properly cited.

This paper is concerned with the nonlinear Klein-Gordon equation with damping term and nonnegative potentials. We introduce a family of potential wells and discuss the invariant sets and vacuum isolating behavior of solutions. Using the potential well argument, we obtain a new existence theorem of global solutions and a blow-up result for solutions in finite time.

\section{Introduction}

In this paper, we consider the nonlinear Klein-Gordon equation with damping term and a real valued potential $T(x)$

$$
\begin{array}{r}
u_{t t}-\Delta u+T(x) u+u+\left|u_{t}\right|^{m-2} u_{t}=|u|^{p-2} u, \\
t>0, \quad x \in R^{n}, \\
u(0, x)=u_{0}, \quad u_{t}(0, x)=u_{1}, \quad x \in R^{n},
\end{array}
$$

where $n \geq 2, m \geq 2$, and

$$
\begin{array}{cc}
2<p<\infty & \text { if } n \leq 2, \\
2<p<\frac{2 n}{n-2} & \text { if } n \geq 3 .
\end{array}
$$

$u=u(t, x)$ is a complex-valued function of $(t, x) \in R^{+} \times R^{n}$, $\Delta$ is the Laplace operator on $R^{n}$, and $u_{t}$ is called the damping term [1]. Ha and Nakagiri [1] studied the local existence for the Cauchy problem (1). Here we are interested in the sharp criteria for global existence and blow-up of solutions of the Cauchy problem (1).

The Klein-Gordon equation is a relativistic version of the Schrödinger equation, which describes relativistic electrons. Levine [2], Ball [3], Payne and Sattinger [4], Zhang [5], and
Gan and Zhang [6] applied the potential well theory and studied the blowing up properties of the nonlinear KleinGordon equations. In [7], Huang and Zhang studied the global existence and blow-up of solutions for the nonlinear Klein-Gordon equation with linear damping term $(m=2)$. In $[8,9]$, the authors studied the existence of global solutions and decay for the energy of solution for the Klein-Gordon equation.

The case of nonlinear damping and source terms $(m>$ $2, p>2$ ) is considered by many authors. For instance, Georgiev and Todorova [10] prove that if $m \geq p$, a global weak solution exists for any initial data; while $2<m<p$ the solution blows up in finite time when the initial energy is sufficiently negative. Ikehata [11] considers the solutions of (1) with small positive initial energy, using the so-called potential well theory introduced by Payne and Sattinger in [4]. Todorova and Vitillaro [12] prove that for any given numbers $\alpha \geq 0, \lambda \geq 0$ there exist infinitely many data $\varphi(x)$, $\psi(x)$ in the energy space such that the initial energy $E(0)=\lambda$, the gradient norm $\|\nabla \varphi\|_{2}=\alpha$, and the solution of (1) blows up in finite time.

In this paper, we consider the interaction between the nonlinear damping and source terms $(m>2, p>2)$ for the Cauchy problem (1). For the local well-posedness of the Cauchy problem (1), the readers may refer to $[13,14]$. We have 
considered the global existence and the finite time blowing up. The potential well theory, which was introduced by Liu [15] and has been used for Schrödinger equations in [16-18], was applied to study the Cauchy problem (1). Based on the results, we show the sharp criteria for global existence and blowing up of its solutions. Applying the perturbed energy method we prove the uniform stabilization for $p \leq m$. And using concavity arguments we prove that the blow-up solutions exist for $p>m$. The results can be extended to the case of more general nonlinearities under suitable assumptions. We extend parts of results in [7] and obtain several new results for system (1).

This paper is organized as follows. In Section 2, a family of potential wells are introduced and a series of properties are given. In Section 3, the invariant sets under the flow of problem (1) and the vacuum isolating behavior of solutions for $0<E(0)<d$ and $E(0) \leq 0$ are discussed. In Section 4, the global existence and blowing up of solutions for problem (1) are proved. In Section 5, the theorem on asymptotic behavior of solutions when $m=2$ is proved.

\section{Potential Wells and Their Properties}

For the Cauchy problem (1), we define the energy space as

$$
H:=\left\{u \in H^{1}\left(R^{n}\right) ; \int_{R^{n}} T(x)\|u\|^{2} d x<\infty\right\} .
$$

$H$ becomes a Hilbert space, continuously embedded in $H^{1}\left(R^{n}\right)$, which is endowed with the inner product

$$
(u, v)_{H}:=\int_{R^{n}}[\nabla u \nabla v+T(x) u v+u v] d x
$$

whose associated norm is denoted by $\|\cdot\|_{H}$.

Throughout this paper, we make the following assumptions on $T(x)$ :

$$
\inf _{x \in R^{n}} T(x)=\bar{T}(x)>0,
$$

$T(x)$ is positive and $a C^{1}$ bounded

$$
\text { measurable function on } R^{n} \text {, }
$$

$$
\lim _{x \rightarrow \infty} T(x)=\infty
$$

We define the energy functions

$$
\begin{array}{r}
E(t)=\frac{1}{2}\left\|u_{t}\right\|_{2}^{2}+\frac{1}{2}\|\nabla u\|_{2}^{2}+\frac{1}{2} T(x)\|u\|_{2}^{2} \\
+\frac{1}{2}\|u\|_{2}^{2}-\frac{1}{p}\|u\|_{p}^{p}, \quad t \in[0, T), \\
E(t)+\int_{0}^{t}\left\|u_{t}\right\|_{m}^{m} d \tau=E(0), \quad t \in[0, T),
\end{array}
$$

and two functionals

$$
\begin{gathered}
S(u)=\frac{1}{2}\|\nabla u\|_{2}^{2}+\frac{1}{2} T(x)\|u\|_{2}^{2}+\frac{1}{2}\|u\|_{2}^{2}-\frac{1}{p}\|u\|_{p}^{p}, \\
R(u)=\|\nabla u\|_{2}^{2}+T(x)\|u\|_{2}^{2}+\|u\|_{2}^{2}-\|u\|_{p}^{p} .
\end{gathered}
$$

Then we define the potential well $W$ as follows:

$$
W \equiv\left\{u \in H\left(R^{n}\right) \mid R(u)>0, S(u)<d_{M}\right\} \cup\{0\},
$$

and the outside set $V$ of the corresponding potential well

$$
V \equiv\left\{u \in H\left(R^{n}\right) \mid R(u)<0, S(u)<d_{M}\right\},
$$

where

$$
\begin{gathered}
d_{M}=\inf _{M} S(u), \\
M=\left\{u \in H\left(R^{n}\right) \backslash\{0\} \mid R(u)=0,\|u\|_{H} \neq 0\right\} .
\end{gathered}
$$

For $\delta>0$, we define

$$
\begin{gathered}
R_{\delta}(u)=\delta\left(\|\nabla u\|_{2}^{2}+T(x)\|u\|_{2}^{2}+\|u\|_{2}^{2}\right)-\|u\|_{p}^{p}, \\
d_{M}(\delta)=\inf _{M_{\delta}} S(u), \\
M_{\delta}=\left\{u \in H\left(R^{n}\right) \backslash\{0\} \mid R_{\delta}(u)=0,\|u\|_{H} \neq 0\right\}, \\
W_{\delta} \equiv\left\{u \in H\left(R^{n}\right) \mid R_{\delta}(u)>0, S(u)<d_{M}(\delta)\right\} \cup\{0\}, \\
0<\delta<\frac{p}{2}, \\
V_{\delta} \equiv\left\{u \in H\left(R^{n}\right) \mid R_{\delta}(u)<0, S(\phi)<d_{M}(\delta)\right\}, \\
0<\delta<\frac{p}{2} .
\end{gathered}
$$

Lemma 1. If $0<\|u\|_{H}<r(\delta)$, then $R_{\delta}(u)>0$. Particularly, if $0<\|u\|_{H}<r(1)$, then $R(u)>0$, where

$$
r(\delta)=\left(\frac{\delta}{C_{*}^{p}}\right)^{1 /(p-2)},
$$

$C_{*}$ is an embedding constant of $H\left(R^{n}\right) \hookrightarrow L^{p}\left(R^{n}\right)$.

Proof. If $0<\|u\|_{H}<r(\delta)$, then

$$
\|u\|_{p}^{p} \leq C_{*}^{p}\|u\|_{H}^{p}=C_{*}^{p}\|u\|_{H}^{p-2}\|u\|_{H}^{2}<\delta\|u\|_{H}^{2} .
$$

It follows that $R_{\delta}(u)>0$.

Lemma 2. If $R_{\delta}(u)<0$, then $\|u\|_{H}>r(\delta)$. Particularly, if $R(u)<0$, then $\|u\|_{H}>r(1)$.

Proof. If $R_{\delta}(u)<0$, then $\|u\|_{H} \neq 0$. From

$$
\delta\|u\|_{H}^{2}<\|u\|_{p}^{p} \leq C_{*}^{p}\|u\|_{H}^{p-2}\|u\|_{H}^{2},
$$

we obtain $\|u\|_{H}>r(\delta)$.

Lemma 3. Assume that (2) holds; then

(1) $d_{M}(\delta) \geq(1 / 2-\delta / p) r(\delta)$ for $0<\delta<p$. In particular, we have $d \geq 2 / \alpha C_{\star}^{\alpha}, \alpha=p /(p-2)$.

(2) $d_{M}(\delta)=\delta^{2 /(p-2)}(1 / 2-\delta / p)(2 p /(p-2)) d$. 
Proof. (1) For $u \in M_{\delta}$, we get $\|u\|_{H}^{2} \geq r(\delta)$ and

$$
\begin{aligned}
S(u) & =\left(\frac{1}{2}-\frac{\delta}{p}\right)\|u\|_{H}^{2}+\frac{1}{p} R_{\delta}(u) \\
& =\left(\frac{1}{2}-\frac{\delta}{p}\right)\|u\|_{H}^{2} \geq\left(\frac{1}{2}-\frac{\delta}{p}\right) r(\delta),
\end{aligned}
$$

which yields $d_{M}(\delta) \geq(1 / 2-\delta / p) r(\delta)$ for $0<\delta<p / 2$.

(2) Let $u \in M$ be a minimizer; that is, $d_{M}=S(u)$. For any $\delta>0$, define $\lambda=\lambda(\delta)$ by

$$
\delta\|\lambda u\|_{H}^{2}=\|\lambda u\|_{p}^{p}
$$

that is,

$$
\delta\|u\|_{H}^{2}=\lambda^{p-2}\|u\|_{p}^{p}
$$

Then, for each $\delta>0$, there exists a unique

$$
\lambda(\delta)=\left(\frac{\delta\|u\|_{H}^{2}}{\|u\|_{p}^{p}}\right)^{1 /(p-2)}
$$

satisfying (18) which implies $\lambda(\delta) u \in M_{\delta}$. Since $u \in M$ implies $\|u\|_{H}^{2}=\|u\|_{p}^{p}$, we get $\lambda(\delta)=\delta^{1 /(p-2)}$. Therefore,

$$
\begin{aligned}
d_{M}(\delta) & \leq S(\lambda(\delta) u) \\
& =\frac{1}{2} \delta^{2 /(p-2)}\|u\|_{H}^{2}-\frac{1}{p} \delta^{p /(p-2)}\|u\|_{p}^{p} \\
& =\left(\frac{1}{2}-\frac{\delta}{p}\right) \delta^{2 /(p-2)}\|u\|_{H}^{2} .
\end{aligned}
$$

Noting that

$$
S(u)=\frac{1}{2}\|u\|_{H}^{2}-\frac{1}{p}\|u\|_{p}^{p}=\frac{p-2}{2 p}\|u\|_{H}^{2}
$$

we have

$$
\begin{aligned}
d_{M}(\delta) & \leq \delta^{2 /(p-2)}\left(\frac{1}{2}-\frac{\delta}{p}\right) \frac{p-2}{2 p} S(u) \\
& =\delta^{2 /(p-2)}\left(\frac{1}{2}-\frac{\delta}{p}\right) \frac{p-2}{2 p} d .
\end{aligned}
$$

On the other hand, let $\delta>0, u \in M_{\delta}$ be a minimizer; that is, $d_{M}(\delta)=S(u)$. It follows that

$$
d_{M}(\delta) \geq \delta^{2 /(p-2)}\left(\frac{1}{2}-\frac{\delta}{p}\right) \frac{p-2}{2 p} d .
$$

Therefore, the conclusion follows from the above discussion.

Lemma 4. Assume that (2) holds; then

(1) $\lim _{\delta \rightarrow 0} d_{M}(\delta)=0, d_{M}(p / 2)=0 . d_{M}(\delta)$ is continuous on $0<\delta \leq p / 2$.

(2) $d_{M}(\delta)$ is increasing on $0<\delta \leq 1$, is decreasing on $1<\delta \leq p / 2$, and takes the maximum $d=d_{M}(1)$ at $\delta=1$.
Proof. From Lemma 3, we obtain

$$
\begin{aligned}
d_{M}^{\prime}(\delta) & =\frac{d}{p-2}\left[(p-2 \delta) \delta^{2 /(p-2)}\right]^{\prime} \\
& =\frac{d}{p-2}\left[(p-2 \delta) \frac{2}{p-2} \delta^{(4-p) /(p-2)}-2 \delta^{2 /(p-2)}\right] \\
& =\frac{2 d p}{(p-2)^{2}} \delta^{(4-p) /(p-2)}(1-\delta) .
\end{aligned}
$$

\section{The Invariant Sets of Solutions}

Lemma 5. Assume that $u_{0} \in H\left(R^{n}\right), u_{1} \in L^{m}\left(R^{n}\right), 0<E(0)<$ $d_{M}, \delta_{1}$, and $\delta_{2}\left(\delta_{1}<\delta_{2}\right)$ are the solutions of the function $d_{M}(\delta)=E(0)$.

(1) If $R\left(u_{0}\right)>0$ or $\left\|u_{0}\right\|_{H}=0$, then $u(t) \in W_{\delta}$ for any $\delta \in\left(\delta_{1}, \delta_{2}\right)$.

(2) If $R\left(u_{0}\right)<0$, then $u(t) \in V_{\delta}$ for any $\delta \in\left(\delta_{1}, \delta_{2}\right)$.

Proof. (1) Let $u(t)$ be any solution of the Cauchy problem (1) with

$$
E(0)=\frac{1}{2}\left\|u_{1}\right\|_{2}^{2}+S\left(u_{0}\right)+\int_{0}^{t}\left\|u_{1}\right\|_{m}^{m} d \tau=d_{M}(\delta)<d_{M},
$$

which gives $S\left(u_{0}\right)<d_{M}$. If $R\left(u_{0}\right)>0$, then from the definition of $W_{\delta}$ we obtain $u_{0}(x) \in W_{\delta}$. If $\left\|u_{0}\right\|_{H}=0$, then $u_{0}(x) \in W_{\delta}$. Therefore $u_{0}(x) \in W_{\delta}, \forall \delta \in\left(\delta_{1}, \delta_{2}\right)$.

Next, we prove $u(t) \in W_{\delta}, \forall \delta \in\left(\delta_{1}, \delta_{2}\right), t \geq 0$. If it is not true, then there must exist a $\widetilde{\delta} \in\left(\delta_{1}, \delta_{2}\right)$ and a $\widetilde{t}>0$ such that $u(\widetilde{t}) \in \partial W_{\tilde{\delta}}$; that is,

$$
R_{\widetilde{\delta}}(u(\widetilde{t}))=0, \quad\left\|u_{0}(\widetilde{t})\right\|_{H} \neq 0, \quad \text { or } S(u(\widetilde{t}))=d_{M}(\widetilde{\delta}) .
$$

From the energy inequality, we have

$$
\begin{array}{r}
\frac{1}{2}\left\|u_{t}\right\|_{2}^{2}+S(u)+\int_{0}^{t}\left\|u_{t}\right\|_{m}^{m} d \tau=E(0)=d_{M}(\delta)<d_{M}, \\
\delta_{1}<\delta<\delta_{2}, \quad t \geq 0 .
\end{array}
$$

Then $S(u(\tilde{t}))=d_{M}$ is impossible. On the other hand, if $R_{\tilde{\delta}}(u(\widetilde{t}))=0,\left\|u_{0}(\widetilde{t})\right\|_{H} \neq 0$, then we obtain $u(\widetilde{t}) \in M$. By the definition of $M$, we have $S(u(\widetilde{t})) \geq d_{M}$, which contradicts (28). Hence $u(t) \in W_{\delta}$ is true.

(2) First we prove $u_{0} \in V_{\delta}$. From the energy inequality

$$
\frac{1}{2}\left\|u_{1}\right\|_{2}^{2}+S\left(u_{0}\right)+\int_{0}^{t}\left\|u_{1}\right\|_{m}^{m} d \tau=E(0)=d_{M}(\delta)<d_{M},
$$

we have

$$
S\left(u_{0}\right)<d_{M}(\delta), \quad \forall \delta_{1}<\delta<\delta_{2} .
$$

Using $R\left(u_{0}\right)<0$ yields $R_{\delta}\left(u_{0}\right)<0$ for $\delta_{1}<\delta<\delta_{2}$. Therefore we obtain $u_{0} \in V_{\delta}$. 
Next, we show that $u(t) \in V_{\delta}$ for $\delta_{1}<\delta<\delta_{2}$ and $t \geq 0$. If it is false, there exist a $\widetilde{\delta} \in\left(\delta_{1}, \delta_{2}\right)$ and a $\widetilde{t}>0$ such that $u(\widetilde{t}) \in$ $\partial V_{\widetilde{\delta}}$; that is,

$$
R_{\widetilde{\delta}}(u(\widetilde{t}))=0, \quad\left\|u_{0}(\widetilde{t})\right\|_{H} \neq 0 \quad \text { or } \quad S(u(\tilde{t}))=d_{M}(\widetilde{\delta}) .
$$

However, from the conservation law we get that $S(u(\widetilde{t}))=$ $d_{M}(\widetilde{\delta})$ is impossible. If $R_{\widetilde{\delta}}(u(\widetilde{t}))=0$, then $R_{\widetilde{\delta}}(u(t))<0$ for $0 \leq t<\tilde{t}$. At the same time, Lemma 2 yields that $\|u(t)\|_{H}>$ $r(\widetilde{\delta})>0,0 \leq t<\widetilde{t}$, and $\|u(\widetilde{t})\|_{H} \geq r(\widetilde{\delta})$. Hence by the definition of $d_{M}(\delta)$, we have $S(u(\widetilde{t})) \geq d_{M}(\widetilde{\delta})$, which contradicts $S(u(t))<d_{M}(\delta)$. So we obtain

$$
u(t) \in V_{\delta}, \quad \forall \delta_{1}<\delta<\delta_{2}, \quad t \geq 0 .
$$

Lemma 6. Assume $u_{0} \in H\left(R^{n}\right), u_{1} \in L^{m}\left(R^{n}\right), 0<E(0)<$ $d_{M}(\delta), \delta_{1}$, and $\delta_{2}\left(\delta_{1}<\delta_{2}\right)$ are the solutions of the function $d_{M}(\delta)=E(0)$. Then $W_{\delta}$ and $V_{\delta}$ are invariant sets under the flow generated by (1), $\forall \delta_{1}<\delta<\delta_{2}$.

Proof. Let $u_{0} \in V_{\delta}$ and $u(t)$ satisfy (1). From (6), (7), and (8), we have

$$
\begin{aligned}
S(u(t)) & \leq E(t) \\
& =E(0)-\int_{0}^{t}\left\|u_{t}\right\|_{m}^{m} d \tau<d_{M}(\delta), \quad t \in[0, T) .
\end{aligned}
$$

To check $u(t) \in V_{\delta}$, we need to prove

$$
R(u(t))<0, \quad t \in[0, T) .
$$

If (34) is not true, by continuity, there would exist a $\bar{t}>0$ such that $R(u(\bar{t}))=0$ because of $R\left(u_{0}\right)<0$. It follows that $u(\bar{t}) \in M_{\delta}$. This is impossible for $S(u(\bar{t}))<d_{M}(\delta)$ and $d_{M}(\delta)=\inf _{u \in M_{\delta}} S(u)$. Thus (34) is true. So $V_{\delta}$ is invariant under the flow generated by (1).

Similarly, we show that $W_{\delta}$ is also invariant under the flow generated by (1). This completes the proof of Lemma 6 .

Lemma 7. Let the initial data $\left(u_{0}, u_{1}\right) \in H\left(R^{n}\right) \times L^{m}\left(R^{n}\right)$, and $u(t, x)$ be a local solution of the Cauchy problem (1) on $[0, T)$. If there exists $a u_{0} \in V_{\delta}$ and $a E(0)<d_{M}(\delta)$, then the inequality

$$
\|\nabla u\|_{2}^{2}+T(x)\|u\|_{2}^{2}+\|u\|_{2}^{2}>\frac{2 p}{p-2} d_{M}(\delta)
$$

is fulfilled for $t \in[0, T)$.

Proof. By the definition of $d_{M}(\delta)$, we have

$$
d_{M}(\delta)=\inf \left\{\frac{p-2}{2 p}\left(\|\nabla u\|_{2}^{2}+T(x)\|u\|_{2}^{2}+\|u\|_{2}^{2}\right)\right\} .
$$

According to Lemma 6, we have $\|u(t, \cdot)\|_{p}^{p}>\|\nabla u(t, \cdot)\|_{2}^{2}$ for $t \in[0, T)$. From (36) and the identity (8), we get

$$
d_{M}(\delta)<\frac{p-2}{2 p}\left(\|\nabla u\|_{2}^{2}+T(x)\|u\|_{2}^{2}+\|u\|_{2}^{2}\right),
$$

which completes the proof of Lemma 7.
In order to extend the case $E(0)>0$ to $E(0) \leq 0$ we give the following lemma.

Lemma 8. Let $u_{0} \in H\left(R^{n}\right), u_{1} \in L^{m}\left(R^{n}\right)$, and (2) hold. Assume $E(0)=0$; then the solutions of problem (1) satisfy

$$
\|u\|_{H}^{2} \geq r_{0}=2\left(\frac{p}{C_{*}^{p}}\right)^{1 /(p-2)} .
$$

Proof. Let $u$ be any solution of problem (1) with $E(0)=0$ and $\left\|u_{0}\right\|_{H} \neq 0, T_{\max }$ the existence time. From (6), (7), and (9), we have

$$
E(t)=\frac{1}{2}\left\|u_{t}\right\|_{2}^{2}+S(u) \leq E(0)=0
$$

and get $S(u) \leq 0$ for $0 \leq t<T_{\max }$. Hence, using

$$
\frac{1}{2}\|u\|_{H}^{2} \leq \frac{1}{p}\|u\|_{p}^{p} \leq C_{*}^{p}\|u\|_{H}^{2}\|u\|_{H}^{p-2}
$$

we see that either $\|u\|_{H}^{2}=0$ or (38) hold. If $\|u\|_{H}^{2}=0$, then $\|u\|_{H}^{2} \equiv 0$ for $0 \leq t<T_{\max }$ (otherwise there exists a $t_{0} \in[0$, $\left.t_{\text {max }}\right)$ such that $\left.0<\left\|u\left(t_{0}\right)\right\|_{H}^{2}<r_{0}\right)$, which contradicts the condition $\left\|u_{0}\right\|_{H} \neq 0$.

Theorem 9. Let $u_{0} \in H\left(R^{n}\right), u_{1} \in L^{m}\left(R^{n}\right), 2<m<p$, and (2) hold. Assume $E(0)<0$ or $E(0)=0$ and $\left\|u_{0}\right\|_{H} \neq 0$. Then the solutions of problem (1) belong to $V_{\delta}$ for $0<\delta<p / 2$.

Proof. Let $u$ be any solution of problem (1) with $E(0)<0$ or $E(0)=0$ and $\left\|u_{0}\right\|_{H} \neq 0, T_{\max }$ the existence time. From (6), we have

$$
\begin{aligned}
E(t)= & \frac{1}{2}\left\|u_{t}\right\|_{2}^{2}+\left(\frac{1}{2}-\frac{\delta}{p}\right)\|u\|_{H}^{2} \\
& +\frac{1}{p} R_{\delta}(u) \leq E(0), \quad 0<\delta<\frac{p}{2},
\end{aligned}
$$

for $0 \leq t<T_{\max }$. From (41) we see that if $E(0)<0$, then $S(u) \leq E(t)<0<d_{M}(\delta)$ for $0 \leq t<T_{\max }$. If $E(0)=0$ and $\left\|u_{0}\right\|_{H} \neq 0$, then by Lemma 8 we obtain $\|u\|_{H}^{2} \geq r_{0}>0$. Therefore, by (41) we have $S(u) \leq E(t) \leq 0<d_{M}(\delta)$ for $0 \leq t<T_{\max }$. Hence, for the above two cases, we have $u \in V_{\delta}$ for $0<\delta<p / 2$.

\section{Sharp Condition for Global Existence and Blow-Up}

Definition 10 (weak solution). The function $u(t, x) \in C([0$, $\left.T) ; H\left(R^{n}\right)\right)$ with $u_{t}(t, x) \in C\left([0, T) ; L^{m}\left(R^{n}\right)\right)$ is called a weak solution of problem $(1)$, such that $u(0, x)=u_{0}(x)$ in $H\left(R^{n}\right)$, $u_{t}(0, x)=u_{1}(x)$ in $L^{m}\left(R^{n}\right)$ and

$$
\begin{aligned}
& \left\langle u_{t t}, v\right\rangle+\int_{R^{n}} \nabla u \cdot \nabla v d x+\int_{R^{n}} T(x) u v d x \\
& \quad+\int_{R^{n}} u v d x+\int_{R^{n}}\left|u_{t}\right|^{m-2} u_{t} v d x=\int_{R^{n}}|u|^{p-2} u v d x,
\end{aligned}
$$

for all $v \in H\left(R^{n}\right)$ and $t \in[0, T)$. 
Theorem 11. Let $\left(u_{0}, u_{1}\right) \in H\left(R^{n}\right) \times L^{m}\left(R^{n}\right), 2<p \leq m$, and (2) hold. Suppose that $0<E(0)<d_{M}, R\left(u_{0}\right)>0$, or $\left\|u_{0}\right\|_{H}=$ 0 ; then Cauchy problem (1) has a global weak solution $u(t, x) \in$ $C\left([0, T) ; H\left(R^{n}\right)\right)\left(u_{t}(t, x) \in C\left([0, T) ; L^{m}\left(R^{n}\right)\right)\right.$ for some $T \in(0, \infty)$ with $u(t) \in W$.

Proof. Let $\left\{w_{j}(x)\right\}$ be a system of base functions in $H\left(R^{n}\right)$. Construct the approximate solution $u_{m}(t, x)$ of problem (1)

$$
u_{m}(t, x)=\sum_{j=1}^{m} g_{j m}(t) w_{j}(x), \quad m=1,2, \ldots,
$$

satisfying

$$
\begin{gathered}
\left(u_{m_{t t}}, w_{s}\right)-\left(\Delta u_{m}, w_{s}\right)+T(x)\left(u_{m}, w_{s}\right) \\
+\left(u_{m}, w_{s}\right)+\left(\left|u_{m_{t}}\right|^{m-2} u_{m_{t}}, w_{s}\right) \\
=\left(\left|u_{m}\right|^{p-2} u_{m}, w_{s}\right), \\
u_{m}(x, 0)=\sum_{j=1}^{m} a_{j m} w_{j}(x) \longrightarrow u_{0}(x) \text { in } H^{1}\left(R^{n}\right), \\
u_{m_{t}}(x, 0)=\sum_{j=1}^{m} b_{j m} w_{j}(x) \longrightarrow u_{1}(x) \text { in } L^{m}\left(R^{n}\right) .
\end{gathered}
$$

Multiplying (44) by $g_{j m}^{\prime}(t)$ and summing for $s$, we have

$$
\frac{d}{d t}\left[\frac{1}{2}\left(\left\|u_{m_{t}}\right\|_{2}^{2}+\left\|u_{m}\right\|_{H}^{2}\right)-\frac{1}{p}\left\|u_{m}\right\|_{p}^{p}\right]+\left\|u_{m_{t}}\right\|_{m}^{m}=0 .
$$

Integrating with respect to $t$, we get

$$
\begin{gathered}
\frac{1}{2}\left(\left\|u_{m_{t}}\right\|_{2}^{2}+\left\|u_{m}\right\|_{H}^{2}\right)-\frac{1}{p}\left\|u_{m}\right\|_{p}^{p}+\int_{0}^{t}\left\|u_{m_{t}}\right\|_{m}^{m} d \tau \\
=\frac{1}{2}\left(\left\|u_{m_{t}}(0)\right\|_{2}^{2}+\left\|u_{m}(0)\right\|_{H}^{2}\right)-\frac{1}{p}\left\|u_{m}(0)\right\|_{p}^{p} .
\end{gathered}
$$

For $E(0)<d_{M}$ and $R\left(u_{0}\right)>0$ or $\|u\|_{H}^{2}=0$, we have

$$
\begin{array}{r}
\frac{1}{2}\left\|u_{m_{t}}\right\|_{2}^{2}+S\left(u_{m}\right)+\int_{0}^{t}\left\|u_{m_{t}}\right\|_{m}^{m} d \tau=E_{m}(0)<d_{M}, \\
0 \leq t<\infty .
\end{array}
$$

From

$$
\begin{array}{r}
\frac{1}{2}\left\|u_{1}\right\|_{2}^{2}+S\left(u_{0}\right)+\int_{0}^{t}\left\|u_{1}\right\|_{m}^{m} d \tau=E(0)<d_{M}, \\
0 \leq t<\infty,
\end{array}
$$

we have $S\left(u_{0}\right)<d_{M}$. Hence from $R\left(u_{0}\right)>0$, we obtain $u_{0} \in$ $W$.

From (45) and (46), for sufficiently large $m$, we obtain

$$
\begin{array}{r}
\frac{1}{2}\left\|u_{m_{t}}\right\|_{2}^{2}+S\left(u_{m}\right)+\int_{0}^{t}\left\|u_{m_{t}}\right\|_{m}^{m} d \tau=E_{m}(0)<d_{M}, \\
0 \leq t<\infty,
\end{array}
$$

and $u_{m}(0) \in W$. Similar with the proof of Lemma 5, from (51), for sufficiently large $m$ and $0 \leq t<\infty$, we can prove $u_{m}(t) \in W$ and

$$
\begin{aligned}
S\left(u_{m}\right) & =\frac{1}{2}\left\|u_{m}\right\|_{H}^{2}-\frac{1}{p}\left\|u_{m}\right\|_{p}^{p} \\
& =\left(\frac{1}{2}-\frac{1}{p}\right)\left\|u_{m}\right\|_{H}^{2}+\frac{1}{p} R\left(u_{m}\right) \geq \frac{p-2}{2 p}\left\|u_{m}\right\|_{H}^{2} .
\end{aligned}
$$

Thus we obtain

$$
\frac{1}{2}\left\|u_{m_{t}}\right\|_{2}^{2}+\frac{p-2}{2 p}\left\|u_{m}\right\|_{H}^{2}<d_{M}, \quad 0 \leq t<\infty ;
$$

then

$$
\begin{gathered}
\left\|u_{m_{t}}\right\|_{2}^{2} \leq 2 d_{M}, \quad 0 \leq t<\infty, \\
\left\|u_{m}\right\|_{H}^{2} \leq \frac{2 p}{p-2} d_{M}, \quad 0 \leq t<\infty, \\
\left\|u_{m}\right\|_{p}^{2} \leq C_{*}^{2}\left\|u_{m}\right\|_{H}^{2} \leq C_{*}^{2} \frac{2 p}{p-2} d_{M}, \quad 0 \leq t<\infty, \\
\left\|\left|u_{m}\right|^{p-2} u_{m}\right\|_{q}^{q}=\left\|u_{m}\right\|_{p}^{p} \leq C_{*}^{p}\left(\frac{2 p}{p-2} d_{M}\right)^{p / 2}, \\
q=\frac{p}{p-1}, \\
0 \leq t<\infty .
\end{gathered}
$$

Using (54) and the method of compact, we obtain that $u(t, x) \in C\left([0, T) ; H\left(R^{n}\right)\right)$ is a global weak solution of problem (1). From Lemma 5, we have $u(t) \in W$ for $0 \leq t<\infty$.

Theorem 12. Let $\left(u_{0}, u_{1}\right) \in H\left(R^{n}\right) \times L^{m}\left(R^{n}\right), 2<p \leq m$, and (2) hold. Assume that $E(0)<d, R_{\delta}\left(u_{0}\right)>0$, or $\left\|u_{0}\right\|_{H}=0$, where $\delta_{1}$ and $\delta_{2}\left(\delta_{1}<\delta_{2}\right)$ are two roots of equation $d_{M}(\delta)=$ $e$. Then the problem (1) admits a unique global solution $u \in$ $C\left([0, T) ; H\left(R^{n}\right)\right)$ and $u \in W_{\delta}$ for $\delta \in\left(\delta_{1}, \delta_{2}\right)$ and $0 \leq t<\infty$.

Proof. From Theorem 11, we see that to prove Theorem 12 we only need to prove $R_{\delta}\left(u_{0}\right)>0$. Indeed, if it is not true, then there exists a $\bar{\delta} \in\left[1, \delta_{2}\right)$ such that $R_{\bar{\delta}}\left(u_{0}\right)=0$. Since $R_{\bar{\delta}}\left(u_{0}\right)>0$ implies $\left\|u_{0}\right\|_{H} \neq 0$, we obtain $S\left(u_{0}\right) \geq d_{M}(\bar{\delta})$, which contradicts $S\left(u_{0}\right) \leq E(0)<d_{M}(\delta)$ for $\delta \in\left(\delta_{1}, \delta_{2}\right)$.

Theorem 13. Let $\left(u_{0}, u_{1}\right) \in H\left(R^{n}\right) \times L^{m}\left(R^{n}\right)$ and (2) hold. Assume $E(0)<d_{M}$.

(1) If $2<m<p$, there exists $t_{0} \in[0, T)$ such that $u\left(t_{0}\right) \in$ $V$; then the solution $u(x, t)$ of Cauchy problem (1) blows up in a finite time.

(2) If $2<p \leq m$, there exists $t_{0} \in[0, T)$ such that $u\left(t_{0}\right) \in W$; then the solution $u(x, t)$ of problem (1) 
globally exists on $[0, \infty)$. Moreover, for $t \in[0, \infty)$, $u(x, t)$ satisfies

$$
\begin{aligned}
& \left\|u_{t}\right\|_{2}^{2}+\frac{p-2}{p} \\
& \quad \times\left(\int\left|\nabla u_{0}\right|^{2} d x+\int T(x)\left|u_{0}\right|^{2} d x+\int\left|u_{0}\right|^{2} d x\right)<2 d_{M} .
\end{aligned}
$$

Proof. By $E(0)<d_{M}$, we have $S\left(u_{0}\right) \leq E(0)<d_{M}$.

Firstly, we prove (1) of Theorem 13. From the energy identity we have

$$
\int_{0}^{t}\left\|u_{t}\right\|_{m}^{m} d s=E(0)-E(t) \leq d_{M},
$$

for all $t \geq 0$.

Denoting $J(t)=\|u(t, \cdot)\|_{2}^{2}$, we have

$$
J^{\prime \prime}(t)=2\left\|u_{t}\right\|_{2}^{2}-2 R(u)-2 \int u u_{t}\left|u_{t}\right|^{m-2} d x .
$$

Using the Hölder inequality and the interpolation inequality, we obtain

$$
\begin{aligned}
\left.\left|\int u u_{t}\right| u_{t}\right|^{m-2} d x \mid & \leq\|u\|_{m}\left\|u_{t}\right\|_{m}^{m-1} \\
& \leq\|u\|_{2}^{\delta}\|u\|_{p}^{1-\delta}\left\|u_{t}\right\|_{m}^{m-1},
\end{aligned}
$$

with $\delta=(1 / m-1 / p) /(1 / 2-1 / p)$. From $R(u)<0$, we have

$$
\int T(x)|u|^{2} d x<\|u\|_{p}^{p}
$$

which together with Lemma 6 give

$$
\begin{aligned}
& \|u\|_{2}^{\delta}\|u\|_{p}^{1-\delta}\left\|u_{t}\right\|_{m}^{m-1} \\
& \quad \leq C\left\|u_{t}\right\|_{m}^{m-1}\|u\|_{p}^{1-p / m-\delta+p \delta / 2}\|u\|_{p}^{p / m} .
\end{aligned}
$$

Using the Young inequality and $1-p / m-\delta+p \delta / 2=0$, we have

$$
\|u\|_{2}^{\delta}\|u\|_{p}^{1-\delta}\left\|u_{t}\right\|_{m}^{m-1} \leq C(\varepsilon)\left\|u_{t}\right\|_{m}^{m}+\varepsilon\|u\|_{p}^{p}
$$

since

$$
\begin{aligned}
-R(u) \geq & -R(u)+\delta(E(t)-E(0)) \\
\geq & \left(1-\frac{\delta}{p}\right)\|u\|_{p}^{p}+\frac{\delta}{2}\left\|u_{t}\right\|_{2}^{2}+\left(\frac{\delta}{2}-1\right) \\
& \times\left(\|\nabla u\|_{2}^{2}+\int T(x)|u|^{2} d x+\|u\|_{2}^{2}\right)-\delta E(0),
\end{aligned}
$$

then

$$
\begin{aligned}
& \frac{1}{2} J^{\prime \prime}(t)+C(\varepsilon)\left\|u_{t}\right\|_{m}^{m} \\
& \geq\left(1+\frac{\delta}{2}\right)\left\|u_{t}\right\|_{2}^{2}+\left(1-\frac{\delta}{p}-\varepsilon\right)\|u\|_{p}^{p} \\
& \quad+\left(\frac{\delta}{2}-1\right)\left(\|\nabla u\|_{2}^{2}+\int T(x)|u|^{2} d x+\|u\|_{2}^{2}\right)-\delta E(0),
\end{aligned}
$$

where the constant $\delta>2$ is chosen as follows.
Since $E(0)<d_{M}$, we choose the constant $\delta$ so that

$$
\frac{2 p d_{M}}{p d_{M}-(p-2) E(0)}<\delta<p .
$$

This guarantees $\delta>2$. Then, using this choice and Lemma 7 we get

$$
\begin{aligned}
& \left(\frac{\delta}{2}-1\right)\left(\|\nabla u\|_{2}^{2}+\int T(x)|u|^{2} d x+\|u\|_{2}^{2}\right)-\delta E(0) \\
& \geq\left(\frac{\delta}{2}-1\right) \frac{2 p}{p-2} d_{M}-\delta E(0) \geq 0 .
\end{aligned}
$$

If the constant $\delta$ is fixed, we choose the constant $\varepsilon$ such that

$$
C_{1}=1-\frac{\delta}{p}-\varepsilon>0 .
$$

Finally, using the inequality (60), (63) and Lemma 7 we have

$$
\begin{aligned}
J^{\prime \prime}(t)+C(\varepsilon)\left\|u_{t}\right\|_{m}^{m} & \geq C_{1}\|u\|_{p}^{p} \\
& \geq C_{1}\left(\|\nabla u\|_{2}^{2}+\int T(x)|u|^{2} d x+\|u\|_{2}^{2}\right) \\
& \geq C_{1} \frac{2 p}{p-2} d_{M},
\end{aligned}
$$

where $C_{1}>0$. Since (56), integrating $(67)$ over $[0, t]$ we have

$$
J^{\prime}(t) \geq C_{1} \frac{p d_{M}}{p-2} t-C(\varepsilon) d_{M}+J^{\prime}(0),
$$

which concludes that there exists a $t_{1}$ such that $\left.J^{\prime}(t)\right|_{t=t_{1}}>0$. Hence, $J(t)$ is increasing for $t>t_{1}$ (which is the interval of existence). Since $R(u)<0$, there exists a $t_{2}$ such that $\|u(t, x)\|_{p}^{p}$ is increasing for $t>t_{2}$. When $t$ is large enough, the quantities $\left\|u_{t}(t, x)\right\|_{m}^{m}$ and $\|\nabla u(t, x)\|_{2}^{2}$ are small enough. Otherwise, assume that there is $t^{*}$ such that $\left\|u_{t}(t, x)\right\|_{m}^{m}>$ $\left\|u_{t}\left(t^{*}, x\right)\right\|_{m}^{m}$ for all $t>t^{*}$. By integrating the inequality, we obtain a contradiction with (56) and $E(t) \geq 0$.

Thus in these cases, the quantity

$$
\begin{aligned}
(1- & \left.\frac{\delta}{p}-\varepsilon\right)\|u\|_{p}^{p}+\left(\frac{\delta}{2}-1\right) \\
& \times\left(\|\nabla u\|_{2}^{2}+\int T(x)|u|^{2} d x+\|u\|_{2}^{2}\right) \\
& -\delta E(0)-C(\varepsilon)\left\|u_{t}\right\|_{m}^{m}
\end{aligned}
$$

will eventually become positive. Therefore for $t$ large enough, from (63) and (65) we have

$$
J^{\prime \prime}(t) \geq\left(1+\frac{\delta}{2}\right)\left\|u_{t}\right\|_{2}^{2} .
$$

Using the Hölder inequality, we get

$$
J(t) J^{\prime \prime}(t) \geq \frac{2+\delta}{8}\left[J^{\prime}(t)\right]^{2} .
$$


Since

$$
\begin{aligned}
& {\left[J^{-(\delta-6) / 8}(t)\right]^{\prime \prime}} \\
& \quad=-\frac{\delta-6}{8} J^{-(\delta+10) / 8}(t)\left[J(t) J^{\prime \prime}(t)-\frac{2+\delta}{8}\left[J^{\prime}(t)\right]^{2}\right],
\end{aligned}
$$

from $(71)$ we have $\left[J^{-(\delta-6) / 8}(t)\right]^{\prime \prime} \leq 0$. Therefore $J^{-(\delta-6) / 8}(t)$ is concave for sufficiently large $t$, and there exists a finite time $T^{*}$ such that

$$
\lim _{t \rightarrow T^{*}} J^{-(\delta-6) / 8}(t)=0 .
$$

From assumption on $T(x)$, we obtain

$$
\int T(x)|u|^{2} d x \geq \bar{T} \int|u|^{2} d x .
$$

Thus one gets $T<\infty$ and

$$
\lim _{t \rightarrow T^{-}}\|u\|_{H}=\infty
$$

We complete the proof of (1) of Theorem 13.

Next, we prove (2) of Theorem 13.

From (6), (7), and (55), we obtain $E(0)<d_{M}$. It follows that $u_{0}$ satisfies

$$
\begin{aligned}
R\left(u_{0}\right)= & \int\left|\nabla u_{0}\right|^{2} d x+\int T(x)\left|u_{0}\right|^{2} d x \\
& +\int\left|u_{0}\right|^{2} d x-\int\left|u_{0}\right|^{p} d x>0
\end{aligned}
$$

which will be proved by contradiction. If (76) is not true, then we have $R\left(u_{0}\right) \leq 0$. Thus there exists $0<\mu \leq 1$ such that $u_{0} \neq 0$ and

$$
\begin{aligned}
R\left(\mu u_{0}\right)= & \mu^{2}\left(\int\left|\nabla u_{0}\right|^{2} d x+\int T(x)\left|u_{0}\right|^{2} d x+\int\left|u_{0}\right|^{2} d x\right) \\
& -\mu^{p} \int\left|u_{0}\right|^{p} d x=0
\end{aligned}
$$

which implies $\mu u_{0} \in M$.

On the other hand, for $0<\mu \leq 1, u_{0} \in W$ and (55) yield

$$
\begin{aligned}
S\left(\mu u_{0}\right)< & \mu^{2} \\
& \times\left(\int\left|\nabla u_{0}\right|^{2} d x+\int T(x)\left|u_{0}\right|^{2} d x+\int\left|u_{0}\right|^{2} d x\right) \\
\leq & d_{M},
\end{aligned}
$$

which is contradictory to Lemma 4.

Therefore, by $R\left(u_{0}\right)>0$ and Lemma 6 , we have $R(u)>0$ and $E(t) \leq E(0)<d_{M}$. Thus

$$
E(t)-\frac{1}{p} S(u) \leq E(0)
$$

namely,

$$
\begin{aligned}
& \frac{1}{2} \int\left|u_{t}\right|^{2} d x+\frac{p-2}{2 p} \\
& \quad \times\left(\int|\nabla u|^{2} d x+\int T(x)|u|^{2} d x+\int|u|^{2} d x\right) \leq E(0) .
\end{aligned}
$$

Therefore we have established the bound of $u(x, t)$ in $H$ for $t \in[0, T)$ and thus the solution $u(x, t)$ of (1) exists globally on $t \in[0, \infty)$.

From (76), $E(0)<d_{M}$, and Lemma 4, we have the estimate (55).

Thus, we complete the proof of Theorem 13.

\section{Asymptotic Behaviour of Solutions}

We now state and prove the following theorem on asymptotic behavior of solutions when $m=2$.

Theorem 14. Let $m=2$ in problem (1). Assume $0<E(0)<$ $d_{m}, R\left(u_{0}\right)>0$, or $\left\|u_{0}\right\|_{H}=0$. For the global solution of the problem (1) given in Theorem 13, we have

$$
E(t) \leq C e^{-k t}, \quad 0 \leq t<\infty,
$$

for some positive constants $C$ and $k$.

Proof. Let $u$ be a global solution of the problem (1); then by Theorem 13, we obtain $u \in W_{\delta}$ for $\delta_{1}<\delta<\delta_{2}$ and $0 \leq t<\infty$, where $\delta_{1}$ and $\delta_{2}\left(\delta_{1}<\delta_{2}\right)$ are two roots of equation $d_{M}(\delta)=$ $E(0)$. Differentiating (7) with respect to $t$ and multiplying the obtained equality by $e^{\gamma t}(\gamma>0)$, we have

$$
\begin{array}{r}
\frac{d}{d t}\left(e^{\gamma t} E(t)\right)+e^{\gamma t}\left\|u_{t}\right\|_{2}^{2}=\gamma e^{\gamma t} E(t), \\
0 \leq t<\infty .
\end{array}
$$

Integrating (82) with respect to $t$, we get

$$
\begin{aligned}
& e^{\gamma t} E(t)+\int_{0}^{t} e^{\gamma \tau}\left\|u_{\tau}\right\|_{2}^{2} d \tau \\
& =E(0)+\gamma \int_{0}^{t} e^{\gamma \tau} E(\tau) d \tau .
\end{aligned}
$$

It follows from $u(t) \in W$ and

$$
E(t)=\frac{1}{2}\left\|u_{t}\right\|_{2}^{2}+\frac{p-2}{2 p}\|u\|_{p}^{p}+\frac{1}{2} R(u) ;
$$

then

$$
E(t) \leq \frac{1}{2}\left\|u_{\tau}\right\|_{2}^{2}+\frac{1}{2} R(u)+\frac{p-2}{2 p}\|u\|_{H}^{2} .
$$

Moreover, taking $v=u$ in (42), we obtain

$$
\frac{d}{d t}\left(u, u_{t}\right)-\left\|u_{t}\right\|_{2}^{2}+\|u\|_{H}^{2}+\frac{1}{2} \frac{d}{d t}\|u\|_{2}^{2}=\|u\|_{p}^{p},
$$


which implies

$$
R(u)=\left\|u_{t}\right\|_{2}^{2}-\frac{d}{d t}\left(u, u_{t}\right)-\frac{1}{2} \frac{d}{d t}\|u\|_{2}^{2}
$$

From (83), (85), and (87), we get

$$
\begin{aligned}
& e^{\gamma \tau} E(\tau) d \tau+\int_{0}^{t} e^{\gamma \tau}\left\|u_{\tau}\right\|_{2}^{2} d \tau \\
& \leq E(0)+\gamma \int_{0}^{t} e^{\gamma \tau}\left(\frac{1}{2}\left\|u_{\tau}\right\|_{2}^{2} d \tau+\frac{1}{2} R(u)+\frac{p-2}{2 p}\|u\|_{H}^{2}\right) d \tau \\
& \leq E(0)+\gamma \int_{0}^{t} e^{\gamma \tau} \\
& \quad \times\left(\frac{1}{2}\left\|u_{\tau}\right\|_{2}^{2} d \tau+\frac{p-2}{2 p}\|u\|_{H}^{2}\right) d \tau \\
& \quad-\frac{\gamma}{4} \int_{0}^{t} e^{\gamma \tau} \frac{d}{d \tau}\left(\left(u, u_{\tau}\right)+\|u\|_{2}^{2}\right) d \tau
\end{aligned}
$$$$
\int_{0}^{t} e^{\gamma \tau} \frac{d}{d \tau}\left(\left(u, u_{t}\right)+\|u\|_{2}^{2}\right) d \tau
$$$$
=2\left(u_{0}, u_{1}\right)+\left\|u_{0}\right\|_{2}^{2}-e^{\gamma t}\left(2\left(u, u_{t}\right)+\|u\|_{2}^{2}\right)
$$$$
+\gamma \int_{0}^{t} e^{\gamma \tau}\left(2\left(u, u_{\tau}\right)+\|u\|_{2}^{2}\right) d \tau
$$$$
\leq 2\left\|u_{0}\right\|_{2}^{2}+\left\|u_{1}\right\|_{2}^{2}+e^{\gamma t}\left(2\|u\|_{2}^{2}+\left\|u_{t}\right\|_{2}^{2}\right)
$$$$
+\gamma \int_{0}^{t} e^{\gamma \tau}\left(2\|u\|_{2}^{2}+\left\|u_{\tau}\right\|_{2}^{2}\right) d \tau .
$$

From (88) and (89), it follows that

$$
\begin{aligned}
& e^{\gamma \tau} E(\tau) d \tau+\int_{0}^{t} e^{\gamma \tau}\left\|u_{\tau}\right\|_{2}^{2} d \tau \\
& \leq E(0)+\gamma \int_{0}^{t} e^{\gamma \tau} \\
& \quad \times\left(\frac{1}{2}\left\|u_{\tau}\right\|_{2}^{2} d \tau+\frac{p-2}{2 p}\|u\|_{H}^{2}\right) d \tau \\
& +\frac{\gamma}{4}\left(2\left\|u_{0}\right\|_{2}^{2}+\left\|u_{1}\right\|_{2}^{2}\right) \\
& +\frac{\gamma}{4} e^{\gamma t}\left(2\|u\|_{2}^{2}+\left\|u_{t}\right\|_{2}^{2}\right) \\
& +\frac{\gamma^{2}}{4} \int_{0}^{t} e^{\gamma \tau}\left(2\|u\|_{2}^{2}+\left\|u_{\tau}\right\|_{2}^{2}\right) d \tau \\
& \leq E(0)+C_{0}+\frac{\gamma}{4} e^{\gamma t}\left(2\|u\|_{2}^{2}+\left\|u_{t}\right\|_{2}^{2}\right) \\
& +\frac{\gamma^{2}}{4} \int_{0}^{t} e^{\gamma \tau}\left\{\left(2+\frac{2(p-2)}{\gamma p}\right)\|u\|_{2}^{2}+\left(1+\frac{\gamma}{4}\right)\left\|u_{\tau}\right\|_{2}^{2}\right\} d \tau,
\end{aligned}
$$

where $C_{0}=(\gamma / 4)\left(2\left\|u_{0}\right\|_{2}^{2}+\left\|u_{1}\right\|_{2}^{2}\right) ;$ then

$$
\begin{aligned}
& e^{\gamma t} E(t) \leq E(0)+C_{0}+\frac{\gamma}{4} e^{\gamma t}\left(2\|u\|_{2}^{2}+\left\|u_{t}\right\|_{2}^{2}\right) \\
&+\frac{\gamma^{2}}{4} \int_{0}^{t} e^{\gamma \tau}\left\{\left(2+\frac{2(p-2)}{\gamma p}\right)\|u\|_{2}^{2}\right. \\
&\left.+\left(1+\frac{4(\gamma-1)}{\gamma^{2}}\right)\left\|u_{\tau}\right\|_{2}^{2}\right\} d \tau .
\end{aligned}
$$

Furthermore, from

$$
\begin{aligned}
E(t) & =\frac{1}{2}\left\|u_{t}\right\|_{2}^{2}+\frac{p-2}{2 p}\|u\|_{H}^{2}+\frac{1}{p} R(u) \\
& >\frac{1}{2}\left\|u_{t}\right\|_{2}^{2}+\frac{p-2}{2 p}\|u\|_{H}^{2}>\frac{p-2}{2 p}\left(\left\|u_{t}\right\|_{2}^{2}+\|u\|_{H}^{2}\right),
\end{aligned}
$$

we obtain

$$
\left\|u_{t}\right\|_{2}^{2}+\|u\|_{H}^{2}<\frac{2 p}{p-2} E(t) .
$$

Let $\delta_{1}=\max \left\{2+(2(p-2)) / \gamma p, 1+(4(\gamma-1)) / \gamma^{2}\right\}$; then

$$
\begin{aligned}
e^{\gamma t} E(t) \leq & E(0)+C_{0}+\frac{2 \gamma}{4} e^{\gamma t}\left(2\|u\|_{H}^{2}+\left\|u_{t}\right\|_{2}^{2}\right) \\
& +\frac{\gamma^{2} \delta_{1}}{4} \int_{0}^{t} e^{\gamma \tau}\left(2\|u\|_{H}^{2}+\left\|u_{t}\right\|_{2}^{2}\right) d \tau \\
\leq & E(0)+C_{0}+\frac{2 \gamma p}{2(p-2)} e^{\gamma t} E(t) \\
& +\frac{\gamma^{2} \delta_{1} p}{2(p-2)} \int_{0}^{t} e^{\gamma \tau} E(\tau) d \tau
\end{aligned}
$$

From (93), we obtain

$$
e^{\gamma t} E(t) \leq C_{1}+C_{2} \int_{0}^{t} e^{\gamma \tau} E(\tau) d \tau,
$$

where $C_{1}=\left(E(0)+C_{0}\right) /(1-2 \gamma p / 2(p-2)), C_{2}=\gamma^{2} \delta_{1} p / 2(p-$ $2)(1-2 \gamma p / 2(p-2))$. Choosing $k$ sufficiently small and together with Gronwall inequality, we have

$$
E(t) \leq C_{1} e^{-k t}
$$

where $k=\gamma\left(1-\gamma \delta_{1} p / 2(p-2)(1-2 \gamma p / 2(p-2))\right)>0$.

\section{Conflict of Interests}

The authors declare that there is no conflict of interests regarding the publication of this paper. 


\section{References}

[1] J. Ha and S. Nakagiri, "Identification problems for the damped Klein-Gordon equations," Journal of Mathematical Analysis and Applications, vol. 289, no. 1, pp. 77-89, 2004.

[2] H. A. Levine, "Instability and nonexistence of global solutions of nonlinear wave equations of the form $P u_{t t}=-A u+F(u)$," Transactions of the American Mathematical Society, vol. 192, pp. 1-12, 1974.

[3] J. M. Ball, "Finite time blow-up in nonlinear problems," in Nonlinear Evolution Equations, pp. 189-205, Academic Press, New York, NY, USA, 1978.

[4] L. E. Payne and D. H. Sattinger, "Saddle points and instability of nonlinear hyperbolic equations," Israel Journal of Mathematics, vol. 22, no. 3-4, pp. 273-303, 1975.

[5] J. Zhang, "Sharp conditions of global existence for nonlinear Schrödinger and Klein-Gordon equations," Nonlinear Analysis: Theory, Methods \& Applications, vol. 48, no. 2, pp. 191-207, 2002.

[6] Z. H. Gan and J. Zhang, "Sharp conditions for global existence for nonlinear Klein-Gordon equations," Acta Mathematica Sinica. Chinese Series, vol. 48, no. 2, pp. 311-318, 2005.

[7] W. Huang and J. Zhang, "Instability of the standing waves for nonlinear Klein-Gordon equations with damping term," Applied Mathematics and Computation, vol. 213, no. 2, pp. 522528, 2009.

[8] T. G. Ha and J. Y. Park, "Global existence and uniform decay of a damped Klein-Gordon equation in a noncylindrical domain," Nonlinear Analysis. Theory, Methods \& Applications, vol. 74, no. 2, pp. 577-584, 2011.

[9] M. Nakao, "Existence of global decaying solutions to the exterior problem for the Klein-Gordon equation with a nonlinear localized dissipation and a derivative nonlinearity," Journal of Differential Equations, vol. 255, no. 11, pp. 3940-3970, 2013.

[10] V. Georgiev and G. Todorova, "Existence of a solution of the wave equation with nonlinear damping and source terms," Journal of Differential Equations, vol. 109, no. 2, pp. 295-308, 1994.

[11] R. Ikehata, "Some remarks on the wave equations with nonlinear damping and source terms," Nonlinear Analysis. Theory, Methods \& Applications, vol. 27, no. 10, pp. 1165-1175, 1996.

[12] G. Todorova and E. Vitillaro, "Blow-up for nonlinear dissipative wave equations in $R^{n}$," Journal of Mathematical Analysis and Applications, vol. 303, no. 1, pp. 242-257, 2005.

[13] F. Gazzola and M. Squassina, "Global solutions and finite time blow up for damped semilinear wave equations," Annales de l'institut Henri Poincaré, vol. 23, no. 2, pp. 185-207, 2006.

[14] S. Lai, "The global weak solution for a generalized CamassaHolm equation," Abstract and Applied Analysis, vol. 2013, Article ID 838302, 6 pages, 2013.

[15] Y. C. Liu, "On potential wells and vacuum isolating of solutions for semilinear wave equations," Journal of Differential Equations, vol. 192, no. 1, pp. 155-169, 2003.

[16] J. Shu and J. Zhang, "Nonlinear Schrödinger equation with harmonic potential," Journal of Mathematical Physics, vol. 47, no. 6, Article ID 063503, 2006.

[17] J. Shu and J. Zhang, "Instability of standing waves for a class of nonlinear Schrödinger equations," Journal of Mathematical Analysis and Applications, vol. 327, no. 2, pp. 878-890, 2007.

[18] J. Shu and J. Zhang, "Sharp conditions of global existence for the generalized Davey-Stewartson system," IMA Journal of Applied Mathematics, vol. 72, no. 1, pp. 36-42, 2007. 




Advances in

Operations Research

mansans

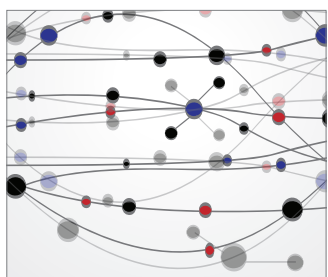

The Scientific World Journal
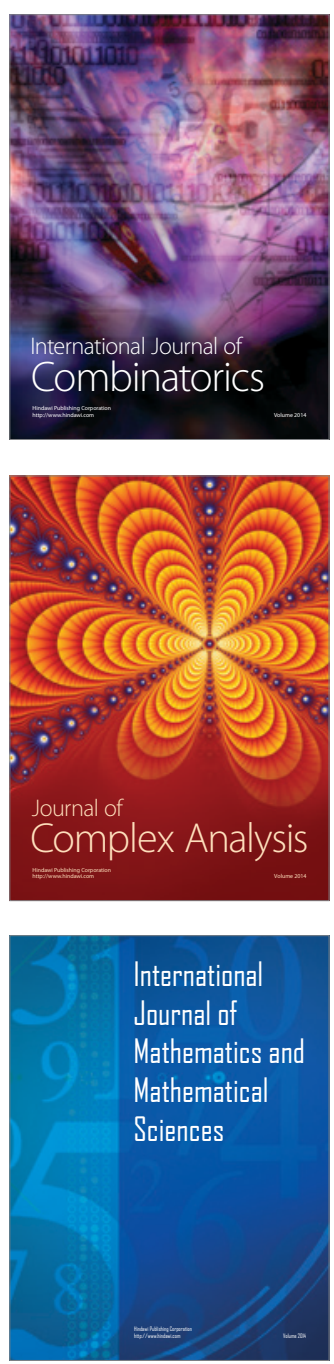
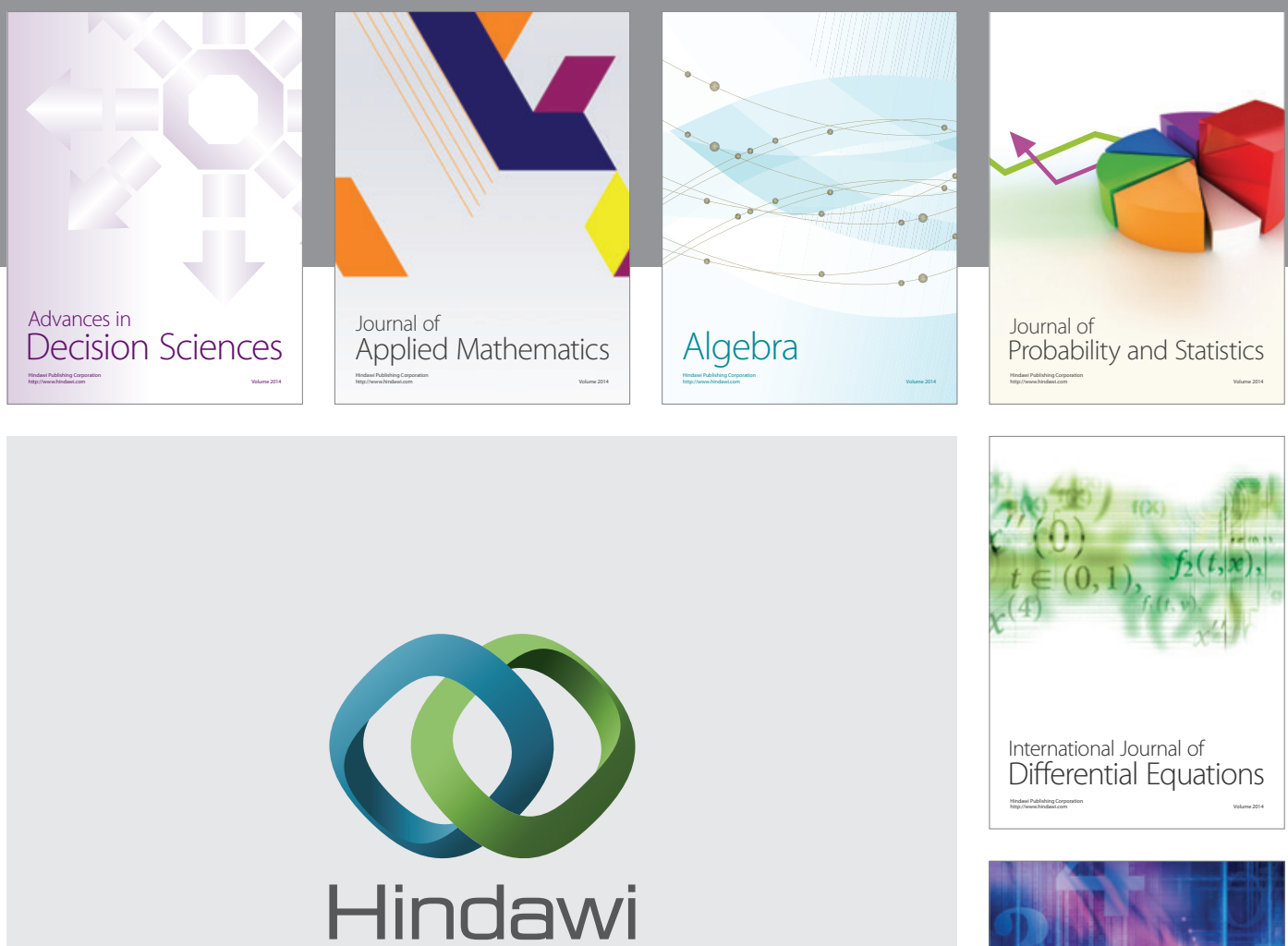

Submit your manuscripts at http://www.hindawi.com


Journal of

Function Spaces

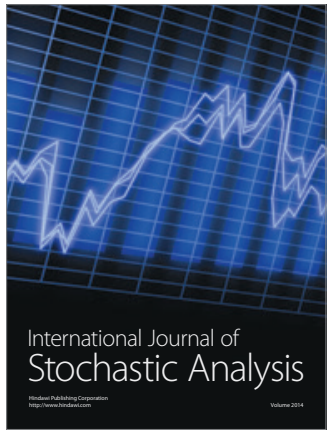

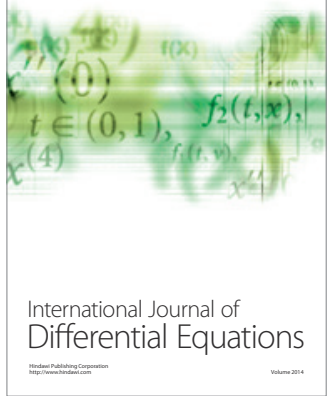
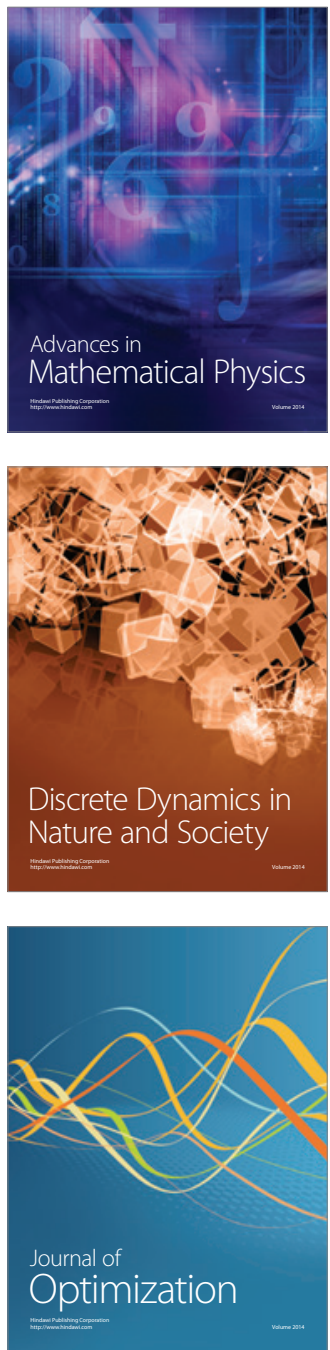